\title{
Roles of the Wada Test and Functional Magnetic Resonance Imaging in Identifying the Language- dominant Hemisphere among Patients with Gliomas Located near Speech Areas
}

\author{
Tatsuya IsHIKAWA, ${ }^{1}$ Yoshihiro MURAGAKI, ${ }^{2}$ Takashi MARUYAMA,,${ }^{1,2}$ \\ Kayoko ABE, ${ }^{3}$ and Takakazu KAWAMATA ${ }^{1}$
}

\begin{abstract}
${ }^{1}$ Department of Neurosurgery, Tokyo Women's Medical University, Tokyo, Japan;
${ }^{2}$ Faculty of Advanced Techno-Surgery, Institute of Advanced Biomedical Engineering and Science, Tokyo Women's Medical University, Tokyo, Japan; ${ }^{3}$ Department of Diagnostic Imaging and Nuclear Medicine, Tokyo Women's Medical University, Tokyo, Japan
\end{abstract}

\begin{abstract}
This study examined the accuracy of functional magnetic resonance imaging (fMRI) in identifying the language-dominant hemisphere and the situations in which the Wada test can be skipped among patients with gliomas located near speech areas. We examined 74 patients [48 men $(64.9 \%)$; mean \pm standard deviation age of $42.7 \pm 13.6$ years (range: 13 to 70 years); 71 right-handed, 2 left-handed, and 1 ambidextrous] with gliomas located near speech areas. All patients underwent the Wada test and fMRI, and 34 patients underwent awake surgery. The "last-and-first" task was administered during fMRI. The Wada test was successful in determining the language-dominant hemisphere in 73 patients (98.6\%): left hemisphere in 68 patients (91.9\%), right hemisphere in 4 patients (5.4\%), and bilateral in 1 patient (1.4\%). The dominant hemisphere for right-handed patients $(\mathrm{n}=71)$ was the left hemisphere in 67 patients $(94.3 \%)$, right hemisphere in 3 patients $(4.2 \%)$, and undetectable in 1 patient $(1.4 \%)$. The fMRI was successful in determining the language-dominant hemisphere in 53 patients $(71.6 \%)$. The results of the Wada test and fMRI were inconsistent in 5 patients (8.6\%), of which $3(5.2 \%)$ exhibited dominance in opposite hemispheres. Furthermore, 2 of these 3 cases $(2.7 \%)$ were contralateral false positive cases, whereby fMRI identified the right-hemisphere as language dominant for right-handed individuals with tumors in the left hemisphere. Based on these findings, we concluded that the Wada test can be skipped if language dominancy can be detected by fMRI.
\end{abstract}

Key words: brain tumor, Wada test, functional magnetic resonance imaging, cortical mapping, endovascular

\section{Introduction}

The extent of glioma resection has a significant influence on the time to progression, 5-year survival, and overall prognosis. Therefore, neurosurgeons strive to remove as much of the lesion as possible. ${ }^{1-5)}$ However, for gliomas located near eloquent areas, preservation of neurological function is essential. Therefore, pre- and peri-operative evaluation of neurological function is necessary for resection of gliomas located near the eloquent areas.

The speech areas are unique among the eloquent areas due to the organization of language dominance within the cerebral hemispheres. The lateralization of

Received February 7, 2016; Accepted September 16, 2016 language dominance is vital in determining surgical strategies and treatment goals. In particular, if the tumor is located in the dominant hemisphere, then awake brain surgery may be considered to facilitate a conservative resection approach that minimizes functional damage.

The Wada test has been broadly applied as a method to determine language-dominant hemispheres. ${ }^{6}{ }^{6}$ Recently, however, functional magnetic resonance imaging (fMRI) has been proposed as a replacement to the Wada test. Here, we investigated both fMRI and the Wada test as pre-operative neurological evaluation approaches for determining language dominance among patients with gliomas located near speech areas. In this study, we retrospectively determined the situations in which the Wada test could be skipped. 


\section{Materials and Methods}

\section{Patients}

The participants were 74 consecutive patients with gliomas located near speech areas who underwent both the Wada test and fMRI. Our patient cohort included 48 men $(64.9 \%)$ and 26 women $(35.1 \%)$ with a mean \pm standard deviation age of $42.7 \pm 13.6$ years (range: 13 to 70 years). Of these patients, 71 were right-handed, 2 were left-handed, and 1 was ambidextrous, which was determined during interviews with the patients. Speech areas near which the gliomas were located included the inferior frontal gyrus, superior temporal gyrus, and the angular gyrus. Speech-related fibers located near the gliomas included the superior longitudinal fasciculus and the inferior fronto-occipital fasciculus. The native language was Japanese for all patients. All patients underwent both the Wada test and fMRI. Thirty-four patients underwent awake brain surgery, based on the results of peri-operative language-dominance detection. According to pathology reports, 39 patients $(52.7 \%)$ had Grade 2 gliomas, 13 patients $(17.6 \%)$ had Grade 3 gliomas, and 22 patients (29.7\%) had Grade 4 gliomas. Tumor location was frontal in 45 patients, insular in 3 patients, parietal in 7 patients, and temporal in 19 patients (Table 1).

Table 1 Demographic and clinical parameters

\begin{tabular}{lc}
\hline Age, years & \\
Median & $42.7 \pm 13.6$ \\
Range & $13-70$ \\
Sex, \%(n) & \\
Male & $64.9(48)$ \\
Female & $35.1(26)$ \\
Grade, \%(n) & \\
Grade 2 & $52.7(39)$ \\
Grade 3 & $17.6(13)$ \\
Grade 4 & $29.7(22)$ \\
Tumor location, \%(n) & \\
Frontal & $60.8(45)$ \\
Insula & $4.1(3)$ \\
Temporal & $25.7(19)$ \\
Parietal & $9.5(7)$ \\
Side, \%(n) & \\
Right & $32.4(24)$ \\
Left & $67.6(50)$ \\
Handed, \%(n) & \\
Right & \\
Left & \\
Both & \\
\hline
\end{tabular}

\section{The Wada test and tasks}

An injection of either sodium amobarbital or sodium thiopental (12.5 mg to $25 \mathrm{mg}$ ) was administered alternately into both internal carotid arteries for the Wada test. We switched to using sodium thiopental following the discontinuation of sodium amobarbital in Japan. Both arms of the patient were elevated prior to injection. The patient was administered language tasks (i.e., repetition, naming, and memory) during the injection phase of each arm. If the patient presented with speech arrest and hemiparesis, then the injected side was judged as the hemisphere of language dominance. If speech arrest occurred following injections in both arms, then language dominance was judged to be bilateral.

\section{fMRI and tasks}

We used EXCELART (Toshiba, 1.5T) machines for fMRI. The percentage of activated voxels in each inferior frontal gyrus was calculated to determine the language-dominant side. Activated voxels were analyzed by a built-in analysis program or the Statistical Parametric Mapping (SPM) 99 program, which performed motion correction and conducted ordinary $t$-tests (values of $P<0.05$ were determined to be statistically significant) to detect the activated voxels. The results were reviewed by neuroradiologists (Fig. 1), and all fMRI results were analyzed in the same way. Patients in the series were administered a "last-andfirst" ("shiritori") brain-activation task during which patients were asked to create a word starting with the last syllable of the last word presented to them. Each task set lasted 30 seconds and was followed by a rest phase for 30 seconds; a total of three sets were administered. Patients who underwent fMRI provided appropriate informed consent consistent with the terms of approval from the internal review board of Tokyo Women's Medical University Hospital.

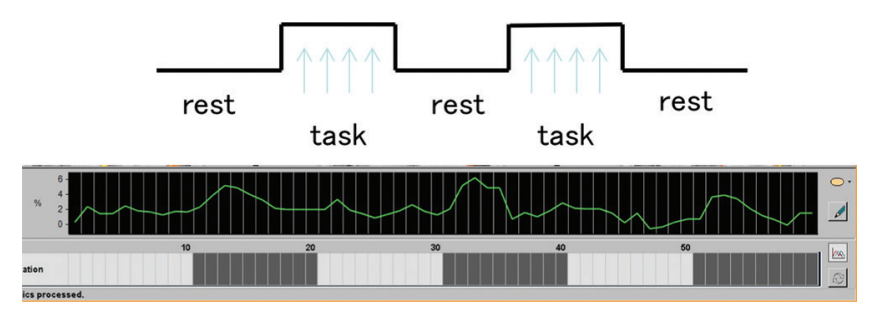

Fig. 1 Patients were administered a brain-activation task in which they were asked to create a word starting with the last syllable of the last word presented to them. Each task set lasted 30 seconds and was followed by a rest phase for 30 seconds; a total of three sets were administered. Activated voxels were analyzed and are displayed relative to the time course. Signal changes were reviewed by neuroradiologists. 


\section{Cortical mapping and tasks}

During awake surgery, naming tasks and verb generation tasks were administered to the patients in order to perform cortical mapping. Pictures were projected onto a monitor, and patients provided the name of each picture for the naming task or stated the verb that matched the action depicted in the pictures for the verb generation task. During the tasks, the exposed cortex was stimulated by an Ojamann's stimulator (Radionics) (50 Hz, $1 \mathrm{~mA}-20 \mathrm{~mA})$. If stimulation to a certain region of the cortex triggered speech arrest, then that region was marked as a speech area.

Determination of the language-dominance hemisphere

If a patient underwent awake surgery, then language dominance was determined by identifying the speech area during the cortical mapping procedure. In contrast, if the patient underwent surgery under general anesthesia, then language dominance was determined by post-operative neurology.

This was a retrospective analysis, based on clinical data from the 74 total patients, and was approved by the Ethics Committee of the Tokyo Women's Medical University (\#3533).

\section{Results}

\section{The Wada test and awake surgery}

Of the 74 patients, the Wada test was successful in determining the hemisphere for language dominance in 73 patients (98.6\%). Sixty-eight $(91.9 \%)$ of 74 patients were left-hemisphere dominant, $4(5.1 \%)$ were righthemisphere dominant, and 1 (1.4\%) exhibited bilateral dominance. One patient became drowsy during the Wada procedure (Table 2), and language dominance could not be determined. Four patients (5.4\%) experienced a seizure during the Wada test. No permanent adverse events associated with the Wada test were observed in any patient. Among the 34 patients who underwent awake surgery, 31 tumors were located in the left hemisphere and 3 were located in the right hemisphere. In all patients whose tumor was in the dominant hemisphere, as determined by the Wada test, the speech area was identified near the location of the tumor during awake surgery. No permanent speech disturbances were observed in any patients who underwent awake surgery. In contrast, patients who underwent general anesthesia had tumors in the non-dominant hemisphere as determined by the Wada test. Of these patients, two presented with transient speech disturbances, which were considered to be caused by dysfunction of the supplementary motor area; no patients exhibited permanent speech disturbances.

\section{fMRI}

Determination of the language-dominant hemisphere was successful in 53 of the 74 patients $(71.6 \%)$ who underwent fMRI. The procedure was unsuccessful for the remaining 21 patients $(28.4 \%)$ due to low or unclear signals. Of these 53 successful patients, the results of the Wada test and fMRI were inconsistent for five patients $(8.6 \%)$. Of these five patients, three patients (5.2\%) exhibited language dominance in the opposite hemispheres between fMRI and the Wada test. Furthermore, two of these three cases $(2.7 \%)$ were "contralateral false positive" cases, wherein the dominant hemisphere was identified as non-dominant. Determining laterality was challenging in some cases due to blurry or unclear voxels (Fig. 2) and/or low intensity signals (Fig. 3). In both of these cases, the patients were

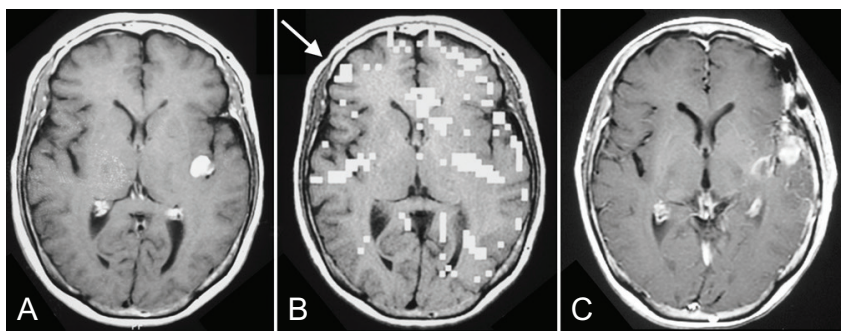

Fig. 2 (A) A 63-year-old right-handed woman with a glioblastoma in the left insular cortex (B) Functional magnetic resonance images in which activated voxels are slightly unclear or blurry in the right inferior frontal gyrus (white arrow). An MRI built-in analysis program revealed language dominance in the right hemisphere. Conversely, the Wada test demonstrated language dominance in the left hemisphere (C) The tumor was removed in an awake state, and no postoperative speech disturbance occurred.

Table 2 Language dominancy according to the Wada test and fMRI

\begin{tabular}{lccccc}
\hline \multirow{2}{*}{ Wada test, \% } & $(n, \mathrm{rt}-\mathrm{t} / \mathrm{lt}-\mathrm{t})$ & \multicolumn{4}{c}{ fMRI, \% $(n, \mathrm{rt}-\mathrm{t} / \mathrm{lt}-\mathrm{t})$} \\
\cline { 3 - 6 } & Right & Left & Bilateral & Unclear \\
\hline Right & $5.4(3 / 1)$ & $2.7(1 / 1)$ & $0.0(0 / 0)$ & $0.0(0 / 0)$ & $2.7(2 / 0)$ \\
Left & $91.9(20 / 48)$ & $4.1(1 / 2)$ & $62.2(11 / 35)$ & $4.0(0 / 2)$ & $23.0(8 / 9)$ \\
Bilateral & $1.4(0 / 1)$ & $0.0(0 / 0)$ & $0.0(0 / 0)$ & $0.0(0 / 0)$ & $1.4(0 / 1)$ \\
Unclear & $1.4(1 / 0)$ & $0.0(0 / 0)$ & $0.0(0 / 0)$ & $0.0(0 / 0)$ & $1.4(1 / 0)$ \\
\hline
\end{tabular}

rt-t: right side tumor, lt-t: left side tumor, fMRI: functional MRI. 
right-handed, the tumor was located in the left hemisphere, the Wada test indicated left-hemisphere dominance, and the fMRI indicated right-hemisphere dominance (Table 2).

\section{Handedness and location of the tumor}

Of the 74 patients, 71 were right-handed, 2 were left-handed, and 1 was ambidextrous. Language

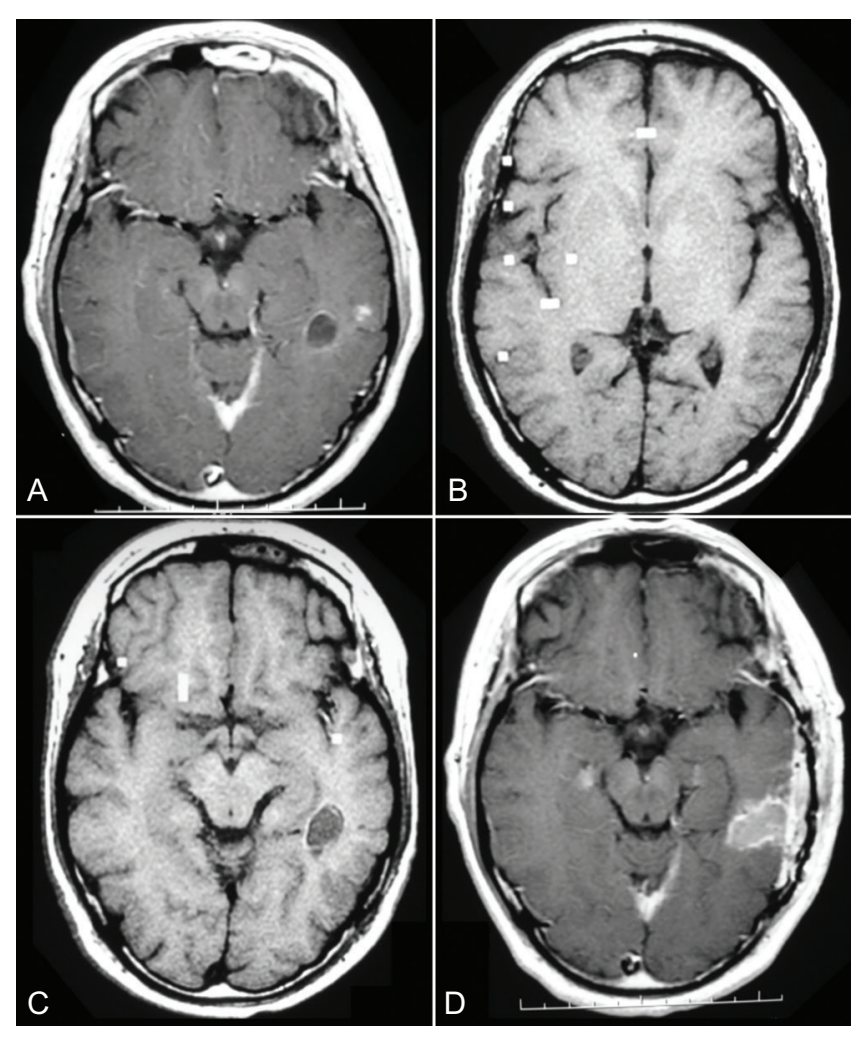

Fig. 3 (A) A 49-year-old right-handed man with a pleomorphic xanthoastrocytoma in the left temporal region. (B, C) Functional magnetic resonance imaging showing low-intensity activated voxels in the right inferior frontal gyrus. An MRI built-in analysis program revealed the right hemisphere to be language dominant. Conversely, the Wada test demonstrated language dominance in the left hemisphere. (D) The tumor was removed while in an awake state, and no post-operative speech disturbance occurred. dominance was observed in the left hemisphere in 68 patients $(91.9 \%)$, right hemisphere in 4 patients $(5.4 \%)$, bilateral in 1 patient $(1.4 \%)$, and undetermined in 1 patient $(1.4 \%)$. The hemisphere that was dominant among right-handed patients (71 patients) was on the left in 67 patients $(94.4 \%)$ and on the right in 3 patients $(4.2 \%$ ) (Table 3 ). For clinical significance, right-handedness with right-dominance was observed in three patients $(4.1 \%)$, and of those patients, tumors in the right hemisphere were observed in one patient $(1.4 \%)$.

\section{Discussion}

Considering the language-dominant hemisphere when planning a surgical strategy for an aggressive removal of a glioma is critical, due to the significant correlation between prognosis and the extent of glioma resection. ${ }^{7,8)}$ At present, the Wada test is the gold standard for determining the languagedominant hemisphere. ${ }^{9-11)}$ However, several studies have reported the use of non-invasive and repeatable language-dominance identification methods, such as magnetoencephalography (MEG) ${ }^{12-14)}$ and fMRI ${ }^{15-25)}$, that could replace the Wada test. In particular, fMRI is widely used, and the advantages of fMRI make it a superior method to the Wada test for patients with glioma. ${ }^{26)}$ The aim of this study was to determine retrospectively the situations in which the Wada test could be skipped.

\section{fMRI: Current problems and future expectations} for gliomas

Of the 74 patients who underwent fMRI, fMRI was unable to accurately detect the languagedominant hemisphere in 21 patients $(26.9 \%)$. In addition, three patients (5.2\%) exhibited paradoxical language dominance. Furthermore, two of these three cases $(2.7 \%)$ were "contralateral false positive" cases, whereby left-hemisphere dominance as determined by the Wada test was identified as right-hemisphere dominance according to fMRI. ${ }^{27)}$ On the other hand, the percentage of activated voxels in "contralateral false positive" cases were

Table 3 Handedness and language dominancy according to the Wada test

\begin{tabular}{lccccc}
\hline \multirow{2}{*}{ Handedness } & Total, \% $(n)$ & \multicolumn{3}{c}{ Dominant side by the Wada test, \% $(n)$} \\
\cline { 3 - 5 } & & Left & Right & Bilateral & Unclear \\
\hline Right & $95.9(71 / 74)$ & $94.4(67 / 71)$ & $4.2(3 / 71)$ & $0.0(0 / 71)$ & $1.4(1 / 71)$ \\
Left & $2.7(2 / 74)$ & $50.0(1 / 2)$ & $50.0(1 / 2)$ & $0.0(0 / 2)$ & $0.0(0 / 2)$ \\
Ambidextrous & $1.3(1 / 74)$ & $0.0(0 / 1)$ & $0.0(0 / 1)$ & $100.0(1 / 1)$ & $0.0(0 / 1)$ \\
Total & & $91.9(68 / 74)$ & $5.4(4 / 74)$ & $1.4(1 / 74)$ & $1.4(1 / 74)$ \\
\hline
\end{tabular}


challenging to determine on the dominant side due to blurry or unclear signals. There are a number of reasons for this phenomenon, one of which is that gliomas can alter the chemical and hemodynamic characteristics of the tumor site and its vicinity compared to normal brain tissue, which results in loss of autoregulation, disturbance of blood circulation by cerebral edema and altered venous return, decreased blood oxygenation leveldependent imaging due to neuronal damage, and other issues. ${ }^{28,29)}$ Another reason is that the tasks generally used for fMRI involve verb generation, and combinations of several tasks are often reported to increase the accuracy of fMRI. ${ }^{30,31)}$ For example, Rutten et al. reported that accuracy as high as $91 \%$ could be achieved using a combination of tasks during fMRI. ${ }^{32)}$ In contrast, our study used only the "last-and-first" task. If patients in our study were administered "matching" tasks or "verb generation" tasks, then the accuracy of the fMRI may have been improved. Furthermore, the combination of multiple procedures may be effective to avoid incorrect judgment. For example, fMRI in combination with MEG may increase the overall accuracy in determining language dominance, particularly in patients with glioma. These non-invasive modalities may eventually replace the Wada test for determining the language-dominant hemisphere.

\section{Handedness and language dominance}

From the results of the Wada test in this study, the left hemisphere was the site of language dominance in $94.3 \%$ of right-handed patients (Table 3). This result is consistent with the results of a previous meta-analysis in which approximately $10 \%$ of righthanded individuals exhibited language dominance in the right hemisphere. Two of our patients $(2.7 \%)$ were "contralateral false positive" cases; both patients were right-handed and had tumors in the left hemisphere. These results suggest that fMRIidentified right-hemisphere language dominance in right-handed individuals with left-hemisphere tumors should be considered "contralateral false positive" results, because right-handed individuals exhibited left-hemisphere language dominance in $94.3 \%$ of cases. In such situations, results from other modalities or information from the Wada test may be needed.

\section{Advantages and disadvantages of the Wada test}

The Wada test involves the possibility of judging both hemispheres as a false-positive ${ }^{33)}$, meaning that both dominant and non-dominant hemispheres would be labeled as dominant due to the effects of anesthesia on the contralateral superior frontal gyrus and supplementary motor area caused by double-filling from the anterior cerebral artery. However, no studies using the Wada test have reported "contralateral false positive" or "false negative" results, as have been reported for fMRI. The most profound problem with the Wada test is its invasiveness. Several patients have suffered from permanent deficits after this test. ${ }^{34)}$ However, the Wada test is reported to cause complications for no more than $0.3 \%$ of simple angiography cases. ${ }^{35)}$ In our own experience, none of the 74 patients in our sample suffered from permanent complications after the Wada test, so we regard this test as fairly safe. In this study, the Wada test determined the language-dominant hemisphere with high sensitivity and specificity. In addition, no patients who underwent extensive tumor removal under general anesthesia from the non-dominant hemisphere according to the Wada test presented with permanent speech disturbance. If there is a situation in which the Wada test is needed, we should not hesitate to perform this test.

\section{When could we skip the Wada test?}

It is clear that the Wada test is the gold standard with extremely high sensitivity and specificity for language dominance determination. All other methods of language dominance determination need to be compared to the Wada test. Patients in whom we should apply the Wada test are those with tumors in the left hemisphere who present with right-hemisphere language dominance according to fMRI. These patients may need extensive surgery under general anesthesia, and the determination of language dominance is a crucial pre-operative step. It is necessary to perform the Wada test to avoid contralateral false positives according to the fMRI. The Wada test also should be performed for cases in which fMRI identifies both hemispheres as language dominant or fails to identify either hemisphere as language dominant. For all other cases, our results indicate that the Wada test can be skipped.

\section{Conclusion}

The current study revealed that the Wada test can be skipped if language dominance is detected by fMRI. The Wada test remains indispensable in some cases, if radical resection under general anesthesia is planned, due to the minor number of contralateral false positive cases. Specifically, for patients with left-hemisphere gliomas, located near speech areas, who are identified as having right-hemisphere language dominance by fMRI. 


\section{Limitations}

This study may not reflect the results of current fMRI, because this study is retrospective and therefore includes old cases.

\section{Acknowledgements}

We would like to thank Dr. Yoshiko Ono at Ebina General Hospital for performing the Wada test and fMRI during the early phase of this study.

\section{Conflicts of Interest Disclosure}

The authors have no financial conflicts of interests. All authors who are members of The Japan Neurological Society have registered online self-reported COI Disclosure Statement Forms through the JNS member website.

\section{References}

1) Committee of Brain Tumor Registry of Japan: Report of Brain Tumor Registry of Japan (1984-2000). Neurol Med Chir (Tokyo) 49: Suppl:PS1-96, 2009

2) Keles GE, Anderson B, Berger MS: The effect of extent of resection on time to tumor progression and survival in patients with glioblastoma multiforme of the cerebral hemisphere. Surg Neurol 52: 371-379, 1999

3) Lacroix M, Abi-Said D, Fourney DR, Gokaslan ZL, Shi W, DeMonte F, Lang FF, McCutcheon IE, Hassenbusch SJ, Holland E, Hess K, Michael C, Miller D, Sawaya R: A multivariate analysis of 416 patients with glioblastoma multiforme: prognosis, extent of resection, and survival. J Neurosurg 95: 190-198, 2001

4) Laws ER, Parney IF, Huang W, Anderson F, Morris AM, Asher A, Lillehei KO, Bernstein M, Brem H, Sloan A, Berger MS, Chang S, Glioma Outcomes Investigators: Survival following surgery and prognostic factors for recently diagnosed malignant glioma: data from the glioma outcomes project. J Neurosurg 99: 467-473, 2003

5) Stummer W, Reulen HJ, Meinel T, Pichlmeier U, Schumacher W, Tonn JC, Rohde V, Oppel F, Turowski B, Woiciechowsky C, Franz K, Pietsch T, ALA-Glioma Study Group: Extent of resection and survival in glioblastoma multiforme: identification of and adjustment for bias. Neurosurgery 62: 564-576, 2008

6) Wada J, Rasmussen T: Intracarotid injection of sodium amytal for the lateralization of cerebral speech dominance. 1960. J Neurosurg 106: 1117-1133, 2007

7) Benzagmout M, Gatignol P, Duffau H: Resection of World Health Organization Grade II gliomas involving Broca's area: methodological and functional considerations. Neurosurgery 61: 741-752, 2007

8) Wu CX, Pu S, Lin Y, Wang YA, Jiang T, Xie J, Wei M, Yi XL, Wang XY: Fractionated resection on low grade gliomas involving Broca's area and insights to brain plasticity. Chin Med J (Engl) 121: 2026-2030, 2008

9) Schevon CA, Carlson C, Zaroff CM, Weiner HJ, Doyle WK, Miles D, Lajoie J, Kuzniecky R, Pacia S, Vazquez B, Luciano D, Najjar S, Devinsky O: Pediatric language mapping: sensitivity of neurostimulation and Wada testing in epilepsy surgery. Epilepsia 48: 539-545, 2007

10) Trenerry MR, Loring DW: Intracarotid amobarbital procedure. The Wada test. Neuroimaging Clin N Am 5: 721-728, 1995

11) Hajek M, Valavanis A, Yonekawa Y, Schiess R, Buck A, Wiesser HG: Selective amobarbital test for the determination of language function in patients with epilepsy with frontal and posterior temporal brain lesions. Epilepsia 39: 389-398, 1998

12) Hirata M, Kato A, Taniguchi M, Saitoh $Y$, Ninomiya H, Ihara A, Kishima H, Oshino S, Baba T, Yorifuji S, Yoshimine T: Determination of language dominance with synthetic aperture magnetometry: comparison with the Wada test. Neuroimage 23: 46-53, 2004

13) Papanicolaou AC, Simos PG, Castillo EM, Breier JI, Sarkari S, Pataraia E, Billingsley RL, Buchanan S, Wheless J, Maggio V, Maggio WW: Magnetocephalography: a noninvasive alternative to the Wada procedure. J Neurosurg 100: 867-876, 2004

14) Kamada K, Takeuchi F, Kuriki S, Todo T, Morita A, Sawamura Y: Dissociated expressive and receptive language functions on magnetoencephalography, functional magnetic resonance imaging, and amobarbital studies. Case report and review of the literature. J Neurosurg 104: 598-607, 2006

15) Lehéricy S, Cohen L, Bazin B, Samson S, Giacomini E, Rougetet R, Hertz-Pannier L, Le Bihan D, Marsault C, Baulac M: Functional MR evaluation of temporal and frontal language dominance compared with the Wada test. Neurology 54: 1625-1633, 2000

16) Spreer J, Arnold S, Quiske A, Wohlfarth R, Ziyeh S, Altenmüller D, Herpers M, Kassubek J, Klisch J, Steinhoff BJ, Honegger J, Schulze-Bonhage A, Schumacher M: Determination of hemisphere dominance for language: comparison of frontal and temporal fMRI activation with intracarotid amytal testing. Neuroradiology 44: 467-474, 2002

17) Yetkin FZ, Swanson S, Fischer M, Akansel G, Morris G, Mueller W, Haughton V: Functional MR of frontal lobe activation: comparison with Wada language results. AJNR Am J Neuroradiol 19: 1095-1098, 1998

18) Binder JR, Swanson SJ, Hammeke TA, Morris GL, Mueller WM, Fischer M, Benbadis S, Frost JA, Rao SM, Haughton VM: Determination of language dominance using functional MRI: a comparison with the Wada test. Neurology 46: 978-984, 1996

19) Deblaere K, Boon PA, Vandemaele P, Tieleman A, Vonck K, Vingerhoets G, Backes W, Defreyne L, Achten E: MRI language dominance assessment in epilepsy patients at 1.0 T: region of interest analysis and comparison with intracarotid amytal testing. Neuroradiology 46: 413-420, 2004 
20) Benson RR, FitzGerald DB, LeSueur LL, Kennedy DN, Kwong KK, Buchbinder BR, Davis TL, Weisskoff RM, Talavage TM, Logan WJ, Cosgrove GR, Belliveau JW, Rosen BR: Language dominance determined by whole brain functional MRI in patients with brain lesions. Neurology 52: 798-809, 1999

21) Spreer J, Quiske A, Altenmüller DM, Arnold S, Schulze-Bonhage A, Steinhoff BJ, Schumacher M: Unsuspected atypical hemispheric dominance for language as determined by fMRI. Epilepsia 42: 957-959, 2001

22) Desmond JE, Sum JM, Wagner AD, Demb JB, Shear PK, Glover GH, Gabrieli JD, Morrell MJ: Functional MRI measurement of language lateralization in Wada-tested patients. Brain 118: 1411-1419, 1995

23) Bahn MM, Lin W, Silbergeld DL, Miller JW, Kuppusamy K, Cook RJ, Hammer G, Wetzel R, Cross $\mathrm{D}$ : Localization of language cortices by functional MR imaging compared with intracarotid amobarbital hemispheric sedation. AJR Am J Roentgenol 169: 575-579, 1997

24) Breier JI, Simos PG, Wheless JW, Constantinou JE, Baumgarthner JE, Venkataraman V, Papanicolaou AC: Language dominance in children as determined by magnetic source imaging and the intracarotid amobarbital procedure: a comparison. J Child Neurol 16: 124-130, 2001

25) Anderson DP, Harvey AS, Saling MM, Anderson V, Kean M, Abbott DF, Wellard RM, Jackson GD: FMRI lateralization of expressive language in children with cerebral lesions. Epilepsia 47: 998-1008, 2006

26) Prothmann S, Puccini S, Dalitz B, Kühn A, Rödel L, Zimmer C, Kahn T: Preoperative mapping of speech-eloquent areas with functional magnetic resonance imaging (fMRI): comparison of different task designs. Rofo 177: 1522-1531, 2005

27) Fujiwara N, Sakatani K, Katayama Y, Murata Y, Hoshino T, Fukaya C, Yamamoto T: Evoked-cerebral blood oxygenation changes in false-negative activations in BOLD contrast functional MRI of patients with brain tumors. Neuroimage 21: 1464-1471, 2004

28) Schreiber A, Hubbe U, Ziyeh S, Hennig J: The influence of gliomas and nonglial space-occupying lesions on blood-oxygen-level-dependent contrast enhancement. AJNR Am J Neuroradiol 21: 1055-1063, 2000

29) Sakatani K, Murata Y, Fujiwara N, Hoshino T, Nakamura S, Kano T, Katayama Y: Comparison of blood-oxygen-level-dependent functional magnetic resonance imaging and near-infrared spectroscopy recording during functional brain activation in patients with stroke and brain tumors. J Biomed Opt 12: 062110, 2007

30) Ruff IM, Petrovich Brennan NM, Peck KK, Hou BL, Tabar V, Brennan CW, Holodny AI: Assessment of the language laterality index in patients with brain tumor using functional MR imaging: effects of thresholding, task selection, and prior surgery. AJNR Am J Neuroradiol 29: 528-535, 2008

31) Rutten GJ, Ramsey NF, van Rijen PC, van Veelen CW: Reproducibility of fMRI-determined language lateralization in individual subjects. Brain Lang 80: 421-437, 2002

32) Rutten GJ, Ramsey NF, van Rijen PC, Alpherts WC, van Veelen CW: FMRI-determined language lateralization in patients with unilateral or mixed language dominance according to the Wada test. Neuroimage 17: 447-460, 2002

33) Lanzenberger R, Wiest G, Geissler A, Barth M, Ringl H, Wöber C, Gartus A, Baumgartner C, Beisteiner R: FMRI reveals functional cortex in a case of inconclusive Wada testing. Clin Neurol Neurosurg 107: 147-151, 2005

34) Jack CR, Nichols DA, Sharbrough FW, Marsh WR, Petersen RC, Hinkeldey NS, Lvnik RJ, Cascino GD, Ilstrup DM: Selective posterior cerebral artery injection of amytal: new method of preoperative memory testing. Mayo Clin Proc 64: 965-975, 1989

35) Dion JE, Gates PC, Fox AJ, Barnett HJ, Blom RJ: Clinical events following neuroangiography: a prospective study. Stroke 18: 997-1004, 1987

Address reprint requests to: Tatsuya Ishikawa, MD, Department of Neurosurgery Tokyo Women's Medical University, 8-1 Kawada-cho, Shinjuku-ku, Tokyo 162-8666, Japan.

e-mail: tishikawa@twmu.ac.jp 